\title{
ON INVERSE NODAL PROBLEM FOR STURM-LIOUVILLE OPERATOR
}

\author{
A.YU. TRYNIN
}

\begin{abstract}
In this paper we propose a solution to a certain inverse Sturm-Liouville problem, which allows one to determine the potential and the boundary conditions of the differential operator on the values of one of the differentials of Gateaux zeroes $x_{k, n}[q] \in(0, \pi)$ of some eigenfunction $\hat{y}\left(x, q, \lambda_{n}[q]\right)$ for an increment $w$ from the set $\mathbb{W}$. As $\mathbb{W}$, we consider some sets of classical and generalized functions.
\end{abstract}

Keywords: eigenfunction of Sturm-Liouville problem, nodal points of Sturm-Liouville problem, Gateaux differential, inverse Sturm-Liouville problem, inverse nodal problem, nodal points.

Mathematics Subject Classification: $34 \mathrm{~A} 55$

\section{INTRODUCTION}

The properties of the eigenfunctions and eigenvalues for Sturm-Liouville problem with nonsmooth potentials is the subject of study of leading scientific schools in spectral theory of differential operators for a rather long period of time. The class of the problems in this field is studied quite well. Not pretending to provide a complete survey in this field, we just mention a series of known works published recently in this scientific direction.

In [1] for a fixed summable potential the authors obtained the asymptotic formulae for the eigenfunctions and the eigenvalues of the classical Sturm-Liouville problem by a modern interpretation of Liouville-Steklov method.

Works [2], 3] were devoted to studying the asymptotics of eigenfunctions and eigenvalues of the Sturm-Liouville operator with a singular potential being a generalized first order eigenfunction $q(x)=u^{\prime}(x)$, where $u \in L_{2}[0, \pi]$.

Works [4], 5] represent the studies where the estimates for the considered characteristics of Sturm-Liouville problem were uniform w.r.t. the potential $q$ in a ball in a Sobolev space.

Paper [6] was devoted to proving the fact that the system of eigenfunctions and the associated functions of a Sturm-Liouville operator with a square summable potential and subject to either periodic or antiperiodic boundary conditions form a Riesz basis.

In [7] there was considered a class of discrete Sturm-Liouville operators for which the essential part of the support of the measure contained a finite number of gaps.

A series of interesting publications [8], [9], [10] was devoted to studying the properties of various types of spectra for periodic self-adjoint second order differential operators on the axis having important applications. One more result of these works was the asymptotic expansions for the eigenvalues and the eigenfunctions of certain perturbed operators.

In fundamental works [11, [12], 13] an analogue of oscillating Sturm theory on distribution of zeroes of eigenfunctions on a spatial net or graphs was constructed.

A.Yu. Trynin, On inverse nodal Problem for Sturm-Liouville operator.

(c) TRYNin A.Yu. 2013.

The work is supported by RFBR (grant no. 13-01-00102).

Submitted April 15, 2013. 
In work [14] there were obtained some asymptotic formulae for the values of differential operators associated with Cauchy problem whose differential expression is a second order linear equation $y^{\prime \prime}+\left[\lambda-q_{\lambda}(x)\right] y=0$, where the potential $q_{\lambda}$ could change subject to $\lambda$, i.e., it was a function of two variables $x$ and $\lambda$. The character of the dependence of the potential $\lambda$ was caused by the fact that for each $\lambda$ function $q_{\lambda}$ belonged to a ball centered at zero and the radius growing slower $\sqrt{\lambda}$ in the space of functions of bounded variations and vanishing at zero. In addition, in [14] the asymptotics for nodal lines of considered differential operators was given under the condition that function $q_{\lambda}$ belongs to the ball centered at zero and with a radius growing slower than $\sqrt{\lambda} / \ln \lambda$. In work [15] an example of potential was constructed which showed that if one neglected the boundedness of variation for $q_{\lambda}$, then the obtained order of asymptotic approximation could be attained neither on the class of functions $q$ in $C[0, \pi]$, for instance, in balls, no even for a particular element in the space of continuous potentials.

Starting from well-known classical works [16]-24] and till the present (see, for instance, papers [4], 25]), a large amount of interesting studies were devoted to the inverse Sturm-Liouville problems, i.e., the problems on constructing Sturm-Liouville operator by some given data. In [16] the author established the equivalence between the formulae $\lambda_{n}=n^{2}, n=0,1,2,3, \ldots$ for the eigenvalues and vanishing of a continuous potential in the Sturm-Liouville problem with Neumann conditions.

Work [17] contains the study of the inverse Sturm-Liouville problem on recovering the parameters of the problem by the spectra. It was shown that in the general case it is impossible to recover Sturm-Liouville operator by one spectrum $\Lambda=\left\{\lambda_{n}\right\}_{n=1}^{\infty}$, while two spectra with the same potential and different boundary conditions are sufficient to determine both the potential and the boundary conditions.

It was proven in [18] that in the case of nonnegative eigenvalues the scattering phase given for all positive energies and any fixed angular momentum determines the potential uniquely.

In [21] methods for recovering a second order differential equation by its spectral function were given. This problem was reduced to a linear integral equation. It was also found out which monotone functions could serve as spectral functions for second order differential equations.

It was shown in work [22] that Sturm-Liouville operator can be uniquely recovered by its spectral function.

In interesting papers [4, 25] an exact uniform asymptotics for the spectral data of SturmLiouville problems was obtained under the assumption that the potential ranges in a ball of a fixed radius in the Sobolev space $W_{2}^{\alpha}[0,1]$ for some $\alpha>-1$. These studies ensures the uniform stability while recovering the potential by the spectral data.

The topical problem on recovering the density of the string by the reaction operator mapping a boundary control into the force applied to an end of the string was considered in work [26].

Beginning from pioneering work [27], in papers [28], [29], [30], [31], [32] there were given uniqueness theorems of nodal problem for various second order differential operator.

A rather wide survey of results obtained for inverse Sturm-Liouville problems can be found in well-known monographs [33], 34], 35].

Let $q \in L[0, \pi]$, and $\lambda_{n}=\lambda_{n}[q]$ be the $n$th eigenvalue of the regular Sturm-Liouville problem (for the definition see [35])

$$
\left\{\begin{array}{c}
\hat{y}^{\prime \prime}+[\lambda-q] \hat{y}=0 \\
\sin \alpha \hat{y}^{\prime}(0)+\cos \alpha \hat{y}(0)=0 \\
\sin \beta \hat{y}^{\prime}(\pi)+\cos \beta \hat{y}(\pi)=0
\end{array}\right.
$$

where $\alpha, \beta \in \mathbb{R}$, and $\hat{y}\left(x, q, \lambda_{n}\right) \equiv \hat{y}_{n}(x)$ are the associated orthonormalized eigenfunctions of this problem $\left\|\hat{y}\left(\cdot, q, \lambda_{n}\right)\right\|_{L_{2}[0, \pi]}=1$. We order the zeroes of function $\hat{y}_{n}$ so that $0 \leqslant x_{0, n}<$ $x_{1, n}<\cdots<x_{n, n} \leqslant \pi$. We fix some $n \in \mathbb{N}$ and $0 \leqslant k \leqslant n, k \in \mathbb{Z}$. By $x_{k, n}[q]$ we denote the functional mapping the potential $q$ into left $k+1$ th left zero of $n$th eigenfunction $\hat{y}\left(x, q, \lambda_{n}[q]\right)$. 
We indicate by

$$
D \phi[q, w]=\lim _{t \rightarrow 0} \frac{\phi(q+t w)-\phi(q)}{t}
$$

the Gâteaux differential of a functional $\phi: L[0, \pi] \rightarrow \mathbb{R}$ with an increment $w \in L[0, \pi]$.

In the case of Dirichlet condition, in [27] there were obtained certain differential relations in terms of Gâteuax differentials for the nodal points of Sturm-Liouville problem. However, these relations involved the derivatives of the eigenfunctions w.r.t. both variable $x$ and the spectral parameter.

Theorem 1 ([27]). Let $q, w \in L_{2}[0, \pi]$, then the Gâteaux differential of functional $x_{k, n}[q]$ $(n \in \mathbb{N}$ and $0 \leqslant k \leqslant n$ as $\alpha=\beta=0$ in (1)) with an increment $w$ satisfies the relation

$$
\begin{aligned}
D x_{k, n}[q, w]= & \frac{1}{\left[y^{\prime}\left(x_{k, n}, q, \lambda_{n}\right)\right]^{2}} \int_{0}^{x_{k, n}} w(\tau) y^{2}\left(\tau, q, \lambda_{n}\right) d \tau \\
& -\frac{\dot{y}\left(x_{k, n}, q, \lambda_{n}\right)}{\dot{y}\left(\pi, q, \lambda_{n}\right) y^{\prime}\left(x_{k, n}, q, \lambda_{n}\right) y^{\prime}\left(\pi, q, \lambda_{n}\right)} \int_{0}^{\pi} w(\tau) y^{2}\left(\tau, q, \lambda_{n}\right) d \tau,
\end{aligned}
$$

where

$$
y^{\prime}(x, q, \lambda)=\frac{\partial}{\partial x} y(x, q, \lambda), \quad \dot{y}(x, q, \lambda)=\frac{\partial}{\partial \lambda} y(x, q, \lambda)
$$

This relation was employed by the author for studying the properties of the inverse nodal Sturm-Liouville problem with a potential in space $L_{2}[0, \pi]$. In [27] the uniqueness theorem was established for recovering the potential by an arbitrary dense in $[0, \pi]$ set of the zeroes of the eigenfunctions.

In work [36] there were provided the differential relations similar to that established in [27]. These relations were provided in terms of Gâteuax differentials for the nodal points of regular Sturm-Liouville problem with a summable potential and Robin boundary conditions and it was necessary to remove Dirichlet conditions $(\alpha \neq \pi l$ and $\beta \neq \pi m, l, m \in \mathbb{Z})$. In particular, by means of these relations, we succeeded in showing the absence of the stability of representing a continuous on $[0, \pi]$ function by interpolation Lagrange processes constructed by the eigenfunctions of Sturm-Liouville problem.

In work 37] there were obtained some differential relations in terms of Gâteuax differentials for the nodal point of regular Sturm-Liouville problem with arbitrary Robin boundary conditions.

Theorem 2 ([37]). Suppose $q, w \in L[0, \pi]$. Then the Gâteaux differential of functional $x_{k, n}[q](n \in \mathbb{N}$ and $0 \leqslant k \leqslant n)$ with an increment $w$ satisfies the relation

$$
D x_{k, n}[q, w]=\frac{1}{\left[\hat{y}^{\prime}\left(x_{k, n}, q, \lambda_{n}\right)\right]^{2}} \int_{0}^{\pi} w(\tau) \hat{y}^{2}\left(\tau, q, \lambda_{n}\right) \beta_{k, n}(\tau) d \tau,
$$

where

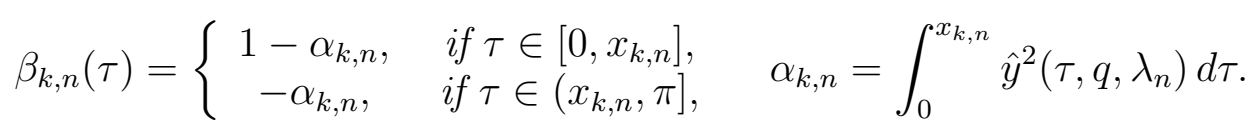

Remark. In the case at least one of the boundary conditions becomes the Dirichlet conition $\alpha=2 \pi l$ or $\beta=2 \pi l, l \in \mathbf{Z}$, i.e., $x_{0, n}[q] \equiv 0$ or $x_{n, n}[q] \equiv \pi$, the associated Gâteaux differential for each $q, w \in L[0, \pi]$ satisfies

$$
D x_{0, n}[q, w]=0 \text { or } D x_{n, n}[q, w]=0 .
$$

In the present work we suggest the solution to some inverse Sturm-Liouville problem allowing one to determine the potential and boundary conditions for a differential operator by the values of Gâteaux differential of one of zeroes $x_{k, n}[q] \in(0, \pi)$ of some eigenfunction $\hat{y}\left(x, q, \lambda_{n}[q]\right)$ with an increment $w$ in set $\mathbb{W}$. In the case $\mathbb{W}=\{\delta[1](x), x \in \mathbb{M}\},(\delta[1](x)$ is the Dirac delta 
function) and $\mathbb{M}$ is dense in $[0, \pi]$, up to then normalization $\int_{0}^{\pi} q(x) d x=0$ we determine the potential of Sturm-Liouville prlblem $q \in L[0, \pi]$ or $q \in C[0, \pi]$. For each fixed $q \in L[0, \pi]$ the values of Gâteaux differentials and of their derivatives w.r.t. $x$ for one of zeroes $x_{k, n}[q] \in$ $(0, \pi)$ of an eigenfunction $\hat{y}\left(x, q, \lambda_{n}[q]\right)$ at the end-points of the segment $[0, \pi]: D x_{k, n}[q, \delta[1](0)]$, $\left.\frac{d D x_{k, n}[q, \delta[1](x)]}{d x}\right|_{x=0}$ and $D x_{k, n}[q, \delta[1](\pi)],\left.\frac{d D x_{k, n}[q, \delta[1](x)]}{d x}\right|_{x=\pi}$ allow us to determine the parameters $\alpha$ and $\beta$ of the boundary conditions in problem $(1)$, respectively. In the case $\mathbb{W}$ is the set of continuously differentiable functions on $[0, \pi]$ with an absolutely continuous derivative, we obtain the uniqueness theorem for solution of the inverse problem under the normalization condition for the potential $\int_{0}^{\pi} q(x) d x=0$. These studies are based on a differential relations obtained in work 37.

The topicality of the studies presented in our work can be demonstrated from the point of view of mathematical physics as follows. We take a non-homogeneous string with an unknown linear density which can have first kind jumps. We suppose that the tension of the string in the state of rest is known. If the initial conditions are so that the vibrations are a standing wave with one of the eigenfrequencies, then the wave nodes are the zeroes of the eigenfunctions associated with this eigenfrequency. The results of the work allow one to find the linear density of the string in the point where mass $\mathbf{m}$ is concentrated by observations of the motion dynamics of one of internals wave nodes during the motion of the point mass $\mathbf{m}$ with a constant speed along the string. Knowledge of Gâteaux differential and of its derivative w.r.t. the indepdendent variable for some internal node of Sturm-Liouville problem under the perturbation of summable potential by the Dirac function on the end-points of the segment gives a possibility to determine constants $\alpha$ and $\beta$ in Robin boundary conditions of problem (1). In its turn, it allows us to determine completely the resisting force for the motion of the string's end-points.

\section{MAIN RESUlts}

Changing the potential $q \in L[0, \pi]$ of problem (1) by an additive constant $q+C$ produces the shift of the spectrum $\Lambda=\left\{\lambda_{n}\right\}_{n=1}^{\infty}$ by the same constant $\left\{\lambda_{n}+C\right\}_{n=1}^{\infty}$. Thus, except explicitly mentioned cases, we assume that the normalization condition

$$
\int_{0}^{\pi} q(x) d x=0
$$

holds true. We define $\delta[f](x)$ which the Dirac delta function (the rigorous justification of the definition can be found, for instance, iun [38, Ch. 2, Sec. 5], [39, §16.7]) as the functional mapping each summable on the segment $[0, \pi]$ function $f$ into the real number

$$
\delta[f](x)=\lim _{\varepsilon \rightarrow 0} \int_{0}^{\pi} f(\tau) \Psi(\tau, x, \varepsilon) d \tau,
$$

where

$$
\begin{gathered}
E(x, \varepsilon)=[x-\varepsilon, x+\varepsilon] \cap[0, \pi], \\
\Psi(\tau, x, \varepsilon)= \begin{cases}\frac{1}{m e s E(x, \varepsilon)}, & \text { as } \tau \in E(x, \varepsilon), \\
0, & \text { as } \tau \in[0, \pi] \backslash E(x, \varepsilon) .\end{cases}
\end{gathered}
$$

We shall denote by

$$
D \phi[q, \delta[1](x)]=\lim _{\varepsilon \rightarrow 0} \lim _{t \rightarrow 0} \frac{\phi(q+t \Psi(\cdot, x, \varepsilon))-\phi(q)}{t}
$$

the action of Gâteaux differential of a functional $\phi$ on an element $q \in L[0, \pi]$ with an increment $\delta[1](x)$. And by

$$
\frac{d^{k} D \phi[q, \delta[1](x)]}{d x^{k}}=\frac{d^{k}}{d x^{k}}\left\{\lim _{\varepsilon \rightarrow 0} \lim _{t \rightarrow 0} \frac{\phi(q+t \Psi(\cdot, x, \varepsilon))-\phi(q)}{t}\right\}
$$


we indicate its $k$ th derivative w.r.t. $x$. Generally speaking, this derivative can be treated as the generalized one [38, Ch. 2, Sec. 6, Subsec. 1]. But in the case when the function $\frac{d^{k}}{d x^{k}}\left\{\lim _{\varepsilon \rightarrow 0} \lim _{t \rightarrow 0} \frac{\phi(q+t \Psi(\cdot, x, \varepsilon))-\phi(q)}{t}\right\}$ is continuous w.r.t. $x$, we assume that the generalized derivative coincides with the classical one in the sense of definitions in 38, Ch. 2, Sec. 5, Items $5,6]$.

Knowing the Gâteaux differential of some internal node of Sturm-Liouville problem under the perturbation of the potential by Dirac function on a dense in $[0, \pi]$ set allows us to determine each summable potential in our problem.

Theorem 3. Let $\mathbb{M}$ be an arbitrary dense in $[0, \pi]$ set,

$$
x_{k, n} \in(0, \pi)
$$

be a zero of an eigenfunction of Sturm-Liouville problem (1) and Gâteaux differential of functional $x_{k, n}[q]$ on an element $q \in L[0, \pi]$ with the increment $\delta[1](x)$ takes value $D x_{k, n}[q, \delta[1](x)]$ in each point $x$ of set $\mathbb{M}$.

Then the potential of Sturm-Liouville problem (1) satisfying the normalization condition (3) can be represented as

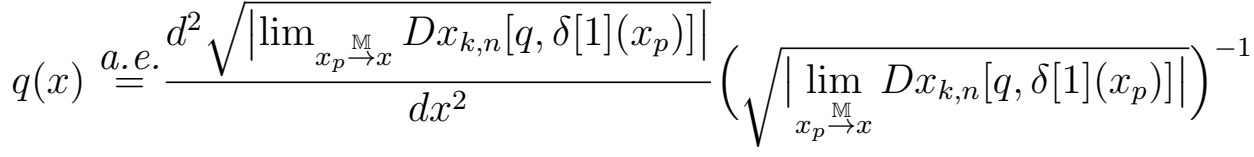

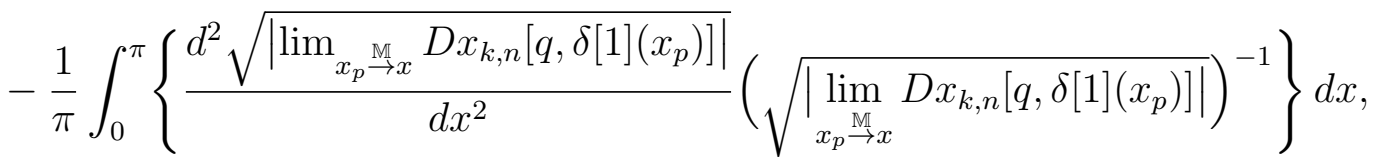

where $\left\{x_{p}\right\}_{p=1}^{\infty}$ is an arbitrary sequence converging to $x$ along set $\mathbb{M}$, i.e., $x_{p} \in \mathbb{M}, x_{p} \rightarrow x$.

Proposition 1. Let (4) be a zero of an eigenfunction to Sturm-Liouville problem (1) and the Gâteaux differential of functional $x_{k, n}[q]$ on an element $q \in L[0, \pi]$ with an increment $\delta[1](x)$ takes the value $D x_{k, n}[q, \delta[1](x)]$ in almost each point $x$ the segment $[0, \pi]$.

Then the potential in Sturm-Liouville problem (1) satisfying normalization condition (3) reads as

$$
\begin{aligned}
q(x) \stackrel{a . e}{=} & \frac{d^{2} \sqrt{\left|D x_{k, n}[q, \delta[1](x)]\right|}}{d x^{2}}\left(\sqrt{\left|D x_{k, n}[q, \delta[1](x)]\right|}\right)^{-1} \\
& -\frac{1}{\pi} \int_{0}^{\pi}\left\{\frac{d^{2} \sqrt{\left|D x_{k, n}[q, \delta[1](x)]\right|}}{d x^{2}}\left(\sqrt{\left|D x_{k, n}[q, \delta[1](x)]\right|}\right)^{-1}\right\} d x .
\end{aligned}
$$

Knowledge of Gâteaux differential and of its derivative w.r.t. the independent variable for some internal node of Sturm-Liouville problem under the perturbation of summable potential by the Dirac function on the end-points of the segment gives a possibility to determine constants $\alpha$ and $\beta$ in Robin boundary conditions of problem (1). It allows us to find completely the resisting force for the motion of the string's end-points.

Proposition 2. Let (4) be a zero of an eigenfunction of Sturm-Liouville problem (1) and the Gâteaux differential of functional $x_{k, n}[q]$ on an element $q \in L[0, \pi]$ with the increment $\delta[1](x)$ and its derivative w.r.t. $x$ take the values $D x_{k, n}[q, \delta[1](0)], D x_{k, n}[q, \delta[1](\pi)],\left.\frac{d D x_{k, n}[q, \delta[1](x)]}{d x}\right|_{x=0}$, and $\left.\frac{d D x_{k, n}[q, \delta[1](x)]}{d x}\right|_{x=\pi}$ on the end-points of the segment $[0, \pi]$. 
Then the parameters of boundary conditions in Sturm-Liouville problem (1) can be found from the relations

$$
\begin{aligned}
& \alpha=\left\{\begin{array}{l}
-\operatorname{arccot}\left\{\left(\left.\frac{d D x_{k, n}[q, \delta[1](x)]}{d x}\right|_{x=0}\right)\left(D x_{k, n}[q, \delta[1](0)]\right)^{-1}\right\}+\pi p, \quad p \in \mathbb{Z}, \\
\text { if } D x_{k, n}[q, \delta[1](0)] \neq 0, \\
\pi p, \quad p \in \mathbb{Z}, \text { if } D x_{k, n}[q, \delta[1](0)]=0,
\end{array}\right. \\
& \beta=\left\{\begin{array}{l}
-\operatorname{arccot}\left\{\left(\left.\frac{d D x_{k, n}[q, \delta[1](x)]}{d x}\right|_{x=\pi}\right)\left(D x_{k, n}[q, \delta[1](\pi)]\right)^{-1}\right\}+\pi r, \quad r \in \mathbb{Z}, \\
\text { if } D x_{k, n}[q, \delta[1](\pi)] \neq 0, \\
\pi r, \quad r \in \mathbb{Z}, \text { if } D x_{k, n}[q, \delta[1](\pi)]=0 .
\end{array}\right.
\end{aligned}
$$

To determine the continuity of potential of Sturm-Liouville problem (1) as well as to determine it everywhere in $[0, \pi]$ we can specify Theorem 3 .

Proposition 3. Let (4) be a zero of an eigenfunction of Sturm-Liouville problem (1) and the Gâteaux differential of functional $x_{k, n}[q]$ on the element $q \in L[0, \pi]$ with the increment $\delta[1](x)$ takes value $D x_{k, n}[q, \delta[1](x)]$ in each point $x$ of a set $\mathbb{M}$ dense in the segment $[0, \pi]$. Then the function

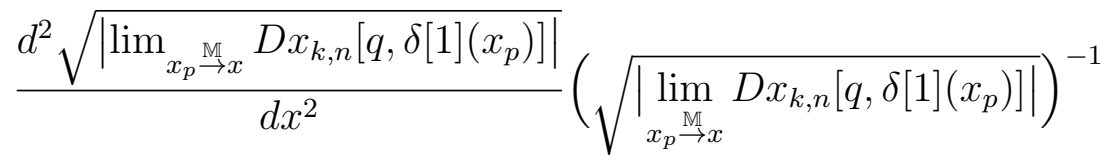

is continuous w.r.t. $x$ on $[0, \pi]$ if and only if the potential of Sturm-Liouville problem (1) is continuous on $[0, \pi]$. And function (5) on $[0, \pi]$ is the potential in Sturm-Liouville problem (1) satisfying normalization condition (3).

Theorem 4. Consider two Sturm-Liouville problems of the form (1) and

$$
\left\{\begin{array}{c}
\hat{\tilde{y}}^{\prime \prime}+[\tilde{\lambda}-\tilde{q}] \hat{\tilde{y}}=0 \\
\sin \tilde{\alpha} \hat{\tilde{y}}^{\prime}(0)+\cos \tilde{\alpha} \hat{\tilde{y}}(0)=0 \\
\sin \tilde{\beta} \hat{\tilde{y}}^{\prime}(\pi)+\cos \tilde{\beta} \hat{\tilde{y}}(\pi)=0
\end{array}\right.
$$

with summable potentials satisfying the normalization conditions (3) and assume that their eigenfunctions $\hat{y}_{n}$ and $\hat{\tilde{y}}_{m} n, m \in \mathbb{N}$ of have a common zero $x^{*}$, i.e., there exist such $0 \leqslant k \leqslant$ $n, 0 \leqslant l \leqslant m, n, m \in \mathbb{N}$ that $x^{*}=x_{k, n}=\tilde{x}_{l, m} \in(0, \pi)$ and the Gâteaux differentials of this zero coincide for the increment $\delta[1](x)$ in each point $x$ of a set $\mathbb{M}$ dense in the segment $[0, \pi]$, i.e.,

$$
D x_{k, n}[q, \delta[1](x)]=D \tilde{x}_{l, m}[\tilde{q}, \delta[1](x)], \text { for each } x \in \mathbb{M} \text {. }
$$

Then $\tilde{q}=q$ a.e. in $[0, \pi], \lambda_{n}=\tilde{\lambda}_{m}$ and $\tilde{\alpha}=\alpha, \tilde{\beta}=\beta$.

By $\mathbb{W}_{\infty}^{1}[0, \pi]$ we denote the set of continuously differentiable and having absolutely continuous derivative on $[0, \pi]$ functions.

Theorem 5. Consider two Sturm-Liouville problems of the form (1) and (8) with summable potentials satisfying the normalization conditions (3) and assume that their eigenfunctions $\hat{y}_{n}$ and $\hat{\tilde{y}}_{m}, n, m \in \mathbb{N}$, have a common zero $x^{*}$, i.e., there exist such $0 \leqslant k \leqslant n, 0 \leqslant l \leqslant m$, $n, m \in \mathbb{N}$ that $x^{*}=x_{k, n}=\tilde{x}_{l, m} \in(0, \pi)$ and the Gâteaux differentials of this zero coincide for each increment $w \in \mathbb{W}_{\infty}^{1}[0, \pi]$,

$$
D x_{k, n}[q, w]=D \tilde{x}_{l, m}[\tilde{q}, w], \text { for each } w \in \mathbb{W}_{\infty}^{1}[0, \pi] .
$$

Then $\tilde{q}=q$ a.e. in $[0, \pi], \lambda_{n}=\tilde{\lambda}_{m}$ and $\tilde{\alpha}=\alpha, \tilde{\beta}=\beta$. 
By $C_{t}^{2}[0, \pi]$ we denote the set of the functions in $C^{1}[0, \pi]$ which are twice continuously differentiable on each of the segments $[0, t]$ and $(t, \pi]$. At the point $t \in[0, \pi]$ the second derivatives of the functions in $C_{t}^{2}[0, \pi]$ can have a jump of the first kind. Classical solutions to Sturm-Liouville problem (1) obey the following

Proposition 4. Let eigenfunctions $\hat{y}_{n}$ and $\hat{\tilde{y}}_{m} n, m \in \mathbb{N}$ of two Sturm-Liouville problems (1) and (8) with continuous potentials satisfying normalization conditions (3) have a common zero, i.e., there exist such $0 \leqslant k \leqslant n, 0 \leqslant l \leqslant m, n, m \in \mathbb{N}$ that $x^{*}=x_{k, n}=\tilde{x}_{l, m} \in(0, \pi)$ and the Gâteaux differentials of this zero coincide for each increment $w \in C_{x^{*}}^{2}[0, \pi]$, i.e.,

$$
D x_{k, n}[q, w]=D \tilde{x}_{l, m}[\tilde{q}, w], \text { for each } w \in C_{x^{*}}^{2}[0, \pi] .
$$

Then $\tilde{q}=q$ everywhere in $[0, \pi], \lambda_{n}=\tilde{\lambda}_{m}$ and $\tilde{\alpha}=\alpha, \tilde{\beta}=\beta$.

The hypothesis of Proposition 1 being a corollary of Theorem 3 is convenient but it employs a redundant information since to recover the potential in Sturm-Liouville problem one has to know the value of $D x_{k, n}[q, \delta[1](x)]$ in almost each point $x$ of segment $[0, \pi]$. The statement of Theorem 3 is non-improvable in the sense that it is impossible to neglect the density of set $\mathbb{M}$ in $[0, \pi]$. This fact is justified by

Proposition 5. For an arbitrary interval $(a, b) \subset[0, \pi]$ there exist two potentials satisfying (3) and having a bounded variation

$$
\stackrel{\stackrel{a . e .}{*} \tilde{q}}{\neq}
$$

such that there exists $n \in \mathbb{N}$ for which the eigenfunctions $\hat{y}_{n}$ and $\hat{\tilde{y}}_{n}$ of two Sturm-Liouville problem (1) and (8) (with $\alpha=\tilde{\alpha}, \beta=\tilde{\beta}$ and potentials $q$ and $\tilde{q}$, respectively) have the same zeros $x_{k, n}[q]=\tilde{x}_{k, n}[\tilde{q}] \in[0, \pi], 0 \leqslant k \leqslant n$, and Gâteaux differentials for each of these zeros coincide for each increment $\delta[1](x)$ in each point $x$ of $[0, \pi] \backslash(a, b)$, i.e.,

$$
\begin{gathered}
D x_{k, n}[q, \delta[1](x)]=D \tilde{x}_{k, n}[\tilde{q}, \delta[1](x)], \\
\text { for eachx } \in[0, \pi] \backslash(a, b), \quad x_{k, n}[q]=\tilde{x}_{k, n}[\tilde{q}] \in[0, \pi], 0 \leqslant k \leqslant n .
\end{gathered}
$$

\section{The PROOF OF MAIN RESUlts}

Proof of Theorem 3. By Theorem 2, for an arbitrary node (4) of Sturm-Liouville problem (1) we have

$$
\begin{aligned}
D x_{k, n}[q, \delta[1](x)] & =\lim _{\varepsilon \rightarrow 0} \frac{1}{\left[\hat{y}^{\prime}\left(x_{k, n}, q, \lambda_{n}\right)\right]^{2}} \int_{0}^{\pi} \Psi(\tau, x, \varepsilon) \hat{y}^{2}\left(\tau, q, \lambda_{n}\right) \beta_{k, n}(\tau) d \tau \\
& =\frac{1}{\left[\hat{y}^{\prime}\left(x_{k, n}, q, \lambda_{n}\right)\right]^{2}} \delta\left[\hat{y}^{2}\left(\cdot, q, \lambda_{n}\right) \beta_{k, n}(\cdot)\right](x) .
\end{aligned}
$$

The function $\hat{y}^{2}\left(\tau, q, \lambda_{n}\right) \beta_{k, n}(\tau)$ is continuously differentiable on $[0, \pi]$. This is why the set $[0, \pi]$ consists of Lebesgue points of the function $\hat{y}^{2}\left(\tau, q, \lambda_{n}\right) \beta_{k, n}(\tau)$ and everywhere in $[0, \pi]$ the identity

$$
D x_{k, n}[q, \delta[1](x)]=\frac{1}{\left[\hat{y}^{\prime}\left(x_{k, n}, q, \lambda_{n}\right)\right]^{2}} \hat{y}^{2}\left(x, q, \lambda_{n}\right) \beta_{k, n}(x)
$$

holds true.

By Theorem 2 and (4) we have $\beta_{k, n}(x) \neq 0$. Thus, on the set $[0, \pi]$ the representation

$$
\left|\hat{y}\left(x, q, \lambda_{n}\right)\right|=\sqrt{\left|D x_{k, n}[q, \delta[1](x)]\right| \frac{\left[\hat{y}^{\prime}\left(x_{k, n}, q, \lambda_{n}\right)\right]^{2}}{\left|\beta_{k, n}(x)\right|}}
$$


holds true. It follows that on each of the segments $\left[0, x_{0, n}\right],\left[x_{0, n}, x_{1, n}\right], \ldots,\left[x_{n-1, n}, x_{n, n}\right],\left[x_{n, n}, \pi\right]$ the eigenfunction can be represented as

$$
\hat{y}\left(x, q, \lambda_{n}\right)=\eta_{l, n} \sqrt{\left|\lim _{x_{p} \rightarrow \mathbb{M} x} D x_{k, n}\left[q, \delta[1]\left(x_{p}\right)\right]\right|}, \quad 0 \leqslant l \leqslant n+1,
$$

where each subscript $l$ of constant $\eta_{l, n}$ corresponds to the index of the segment in which $x$ is located and $\left\{x_{p}\right\}_{p=1}^{\infty}$ is an arbitrary sequence converging to $x$ along set $\mathbb{M}$ dense in the segment $[0, \pi]$, i.e., $x_{p} \in \mathbb{M}$. By the definition [40, Ch. IV, Sec. 1] of a generalized solution to the differential equation in Sturm-Liouville problem (1), on each segment $\left[0, x_{0, n}\right],\left[x_{0, n}, x_{1, n}\right]$, $\ldots,\left[x_{n-1, n}, x_{n, n}\right],\left[x_{n, n}, \pi\right]$ the function $y\left(x, q, \lambda_{n}\right)$ has an absolutely continuous and almost everywhere the second derivative. After the substitution in the equation in (1), this second derivative transforms the equation into the identity almost everywhere. It implies the existence of the second derivative

$$
\frac{d^{2} \sqrt{\left|\lim _{x_{p} \underset{\mathbb{M}}{\mathrm{M}} x} D x_{k, n}\left[q, \delta[1]\left(x_{p}\right)\right]\right|}}{d x^{2}}
$$

for almost each $x \in[0, \pi]$ as well as the representation

$$
\begin{aligned}
q(x)-\lambda_{n} & \stackrel{\text { a.e. }}{=} \frac{\hat{y}^{\prime \prime}\left(x, q, \lambda_{n}\right)}{\hat{y}\left(x, q, \lambda_{n}\right)} \\
& \stackrel{\text { a.e. }}{ } \frac{d^{2} \sqrt{\left|\lim _{x_{p} \mathbb{M}_{x}} D x_{k, n}\left[q, \delta[1]\left(x_{p}\right)\right]\right|}}{d x^{2}}\left(\sqrt{\left|\lim _{x_{p} \rightarrow x} D x_{k, n}\left[q, \delta[1]\left(x_{p}\right)\right]\right|}\right)^{-1} .
\end{aligned}
$$

Thus, the potential in Sturm-Liouville problem (1) satisfying normalization condition (3) can be represented via the values of Gâteaux differential with the increment being Dirac delta function as

$$
\begin{aligned}
& q(x) \stackrel{\text { a.e. }}{=} \frac{d^{2} \sqrt{\left|\lim _{x_{p} \rightarrow \mathbb{M} x} D x_{k, n}\left[q, \delta[1]\left(x_{p}\right)\right]\right|}}{d x^{2}}\left(\sqrt{\left|\lim _{x_{p} \rightarrow \mathbb{M} x} D x_{k, n}\left[q, \delta[1]\left(x_{p}\right)\right]\right|}\right)^{-1} \\
& -\frac{1}{\pi} \int_{0}^{\pi}\left\{\frac{d^{2} \sqrt{\left|\lim _{x_{p} \mathbb{M} x} D x_{k, n}\left[q, \delta[1]\left(x_{p}\right)\right]\right|}}{d x^{2}}\left(\sqrt{\left|\lim _{x_{p} \rightarrow x} D x_{k, n}\left[q, \delta[1]\left(x_{p}\right)\right]\right|}\right)^{-1}\right\} d x .
\end{aligned}
$$

The proof is complete.

Proof of Proposition 1. The desired statement follows immediately from Theorem 3 if as $\mathbb{M}$ one takes the segment $[0, \pi]$, while sequence $\left\{x_{p}\right\}_{p=1}^{\infty}$ is to be taken stationary.

Proof of Proposition 2. By the definition [40, Ch. IV, Sec. 1] of a generalized solution to the differential equation in Sturm-Liouville problem (1), on each of segments $\left[0, x_{0, n}\right],\left[x_{n, n}, \pi\right]$ the function $y\left(x, q, \lambda_{n}\right)$ has an absolute continuous derivative. The representation (14) yields also the existence of $\frac{d \sqrt{\left|D x_{k, n}[q, \delta[1](x)]\right|}}{d x}$ on the end-points of the segment $[0, \pi]$ and the possibility of recovering the parameters of boundary conditions in Sturm-Liouville problem (1) by formulae (7). The proof is complete.

Remark. In particular, it follows from representation $(13)$ and theorem 2 that as $x<x_{k, n}$, $D x_{k, n}[q, \delta[1](x)] \geqslant 0$, while $D x_{k, n}[q, \delta[1](x)] \leqslant 0$ for $x>x_{k, n}$. If we perturb the potential in a node by Dirac function, then this node and other nodes remain unchanged. Indeed, it follows from (13) that

$$
D x_{k, n}\left[q, \delta[1]\left(x_{l, n}\right)\right] \frac{\left[\hat{y}^{\prime}\left(x_{k, n}, q, \lambda_{n}\right)\right]^{2}}{\beta_{k, n}\left(x_{l, n}\right)}=\hat{y}^{2}\left(x_{l, n}, q, \lambda_{n}\right)=0, \quad k, l \in[0, n] .
$$


Proof of Proposition 3. The continuity of function (15) in (14) implies the validity of the identity in (15) everywhere in $[0, \pi]$. The proof is complete.

Remark. Under the hypothesis of Theorem 3, for a potential normalized by relation (3), the $n$th eigenvalue of problem (1) is determined by the formula

$$
\lambda_{n}=-\frac{1}{\pi} \int_{0}^{\pi}\left\{\frac{d^{2} \sqrt{\left|\lim _{x_{p} \rightarrow x} D x_{k, n}\left[q, \delta[1]\left(x_{p}\right)\right]\right|}}{d x^{2}}\left(\sqrt{\left|\lim _{x_{p} \rightarrow x} D x_{k, n}\left[q, \delta[1]\left(x_{p}\right)\right]\right|}\right)^{-1}\right\} d x .
$$

The values of Gâteaux differential allow us to calculate not only the eigenvalue but also to determine the eigenfunction $\hat{y}\left(x, q, \lambda_{n}\right)$. In order to do it, we need to choose appropriate constants $\eta_{l, n}$ in representation (14).

Proof of Theorem 4. By (9) and (13), for each $x \in \mathbb{M}$ we have the identity

$$
\begin{aligned}
\frac{1}{\left[\hat{y}^{\prime}\left(x_{k, n}, q, \lambda_{n}\right)\right]^{2}} \hat{y}^{2}\left(x, q, \lambda_{n}\right) \beta_{k, n}(x) & =D x_{k, n}[q, \delta[1](x)] \\
& =D \tilde{x}_{l, m}[\tilde{q}, \delta[1](x)]=\frac{1}{\left[\hat{\tilde{y}}^{\prime}\left(\tilde{x}_{l, m}, \tilde{q}, \tilde{\lambda}_{m}\right)\right]^{2}} \hat{\tilde{y}}^{2}\left(\tilde{x}, \tilde{q}, \tilde{\lambda}_{m}\right) \tilde{\beta}_{l, m}(x) .
\end{aligned}
$$

It yields that the sets of the nodes of considered problems coincide and for each of the segments $\left[0, x_{0, n}\right],\left[x_{0, n}, x_{1, n}\right], \ldots,\left[x_{n-1, n}, x_{n, n}\right],\left[x_{n, n}, \pi\right]$ the eigenfunctions can be represented as

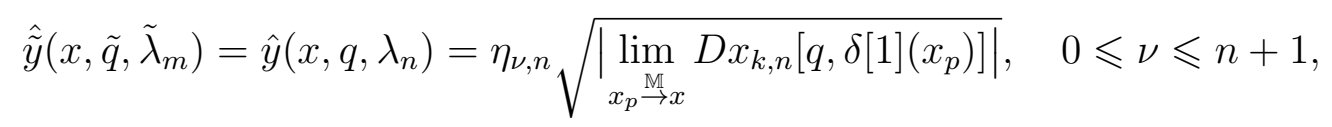

where each subscript $\nu$ of constant $\eta_{\nu, n}$ corresponds to the index of the segment in which $x$ is located and $\left\{x_{p}\right\}_{p=1}^{\infty}$ is an arbitrary sequence converging to $x$ along set $\mathbb{M}$ dense in the segment $[0, \pi]$ set, i.e., $x_{p} \in \mathbb{M}$. Then representation 15 implies

$$
\tilde{q}-\tilde{\lambda}_{m}=q-\lambda_{n}
$$

almost everywhere in $[0, \pi]$. Integrating the obtained relation w.r.t. $\tau$ from 0 to $\pi$ and taking into consideration the normalization condition (3), we get $\lambda_{n}=\tilde{\lambda}_{m}$. Identities (16) yield also $\tilde{\alpha}=\alpha$ and $\tilde{\beta}=\beta$.

Remark. In the same way one can show that a zero $x_{k, n}[q]$ (4) of an eigenfunction of SturmLiouville problem (1) and values $D x_{k, n}[q, \delta[1](x)]$ of Gâteaux differential of functional $x_{k, n}[q]$ on element $q \in C[0, \pi]$ with the increment $\delta[1](x)$ in each point $x$ of a dense in segment $[0, \pi]$ set determines uniquely the potential $q \in C[0, \pi]$ up to the normalization (3).

Proof of Theorem 5. Suppose for some $0 \leqslant k \leqslant n, 0 \leqslant l \leqslant m, n, m \in \mathbb{N} x^{*}=x_{k, n}=\tilde{x}_{l, m} \in$ $(0, \pi)$ is a common zero for considered in Theorem 5 Sturm-Liouville problems, then by Theorem 2 and 10 for each $w \in \mathbb{W}_{\infty}^{1}[0, \pi]$ we have the identity

$$
\begin{aligned}
0 & =D x_{k, n}[q, w]-D \tilde{x}_{l, m}[\tilde{q}, w] \\
& =\int_{0}^{\pi} w(\tau)\left\{\frac{1}{\left[\hat{y}^{\prime}\left(x^{*}, q, \lambda_{n}\right)\right]^{2}} \hat{y}^{2}\left(\tau, q, \lambda_{n}\right) \beta_{k, n}(\tau)-\frac{1}{\left[\hat{\tilde{y}}^{\prime}\left(x^{*}, \tilde{q}, \tilde{\lambda}_{m}\right)\right]^{2}} \hat{\tilde{y}}^{2}\left(\tau, \tilde{q}, \tilde{\lambda}_{m}\right) \tilde{\beta}_{l, m}(\tau)\right\} d \tau
\end{aligned}
$$

By (2), the function

$$
\frac{1}{\left[\hat{y}^{\prime}\left(x^{*}, q, \lambda_{n}\right)\right]^{2}} \hat{y}^{2}\left(\tau, q, \lambda_{n}\right) \beta_{k, n}(\tau)-\frac{1}{\left[\hat{\tilde{y}}^{\prime}\left(x^{*}, \tilde{q}, \tilde{\lambda}_{m}\right)\right]^{2}} \hat{\tilde{y}}^{2}\left(\tau, \tilde{q}, \tilde{\lambda}_{m}\right) \tilde{\beta}_{l, m}(\tau)
$$

belongs to the set $\mathbb{W}_{\infty}^{1}[0, \pi]$. Taking the function

$$
w(\tau)=\frac{1}{\left[\hat{y}^{\prime}\left(x^{*}, q, \lambda_{n}\right)\right]^{2}} \hat{y}^{2}\left(\tau, q, \lambda_{n}\right) \beta_{k, n}(\tau)-\frac{1}{\left[\hat{\tilde{y}}^{\prime}\left(x^{*}, \tilde{q}, \tilde{\lambda}_{m}\right)\right]^{2}} \hat{\tilde{y}}^{2}\left(\tau, \tilde{q}, \tilde{\lambda}_{m}\right) \tilde{\beta}_{l, m}(\tau),
$$


as the increment for both Gâteaux differentials, by (17) we obtain

$$
\int_{0}^{\pi}\left\{\frac{1}{\left[\hat{y}^{\prime}\left(x^{*}, q, \lambda_{n}\right)\right]^{2}} \hat{y}^{2}\left(\tau, q, \lambda_{n}\right) \beta_{k, n}(\tau)-\frac{1}{\left[\hat{\tilde{y}}^{\prime}\left(x^{*}, \tilde{q}, \tilde{\lambda}_{m}\right)\right]^{2}} \hat{\tilde{y}}^{2}\left(\tau, \tilde{q}, \tilde{\lambda}_{m}\right) \tilde{\beta}_{l, m}(\tau)\right\}^{2} d \tau=0 .
$$

Since the integrand is nonnegative, by Theorem 2 we have the representation

$$
\hat{\tilde{y}}\left(\tau, \tilde{q}, \tilde{\lambda}_{m}\right) \stackrel{\text { a.e. }}{=}\left\{\begin{array}{ll}
C_{1} \hat{y}\left(\tau, q, \lambda_{n}\right), & \text { as } \tau \in\left[0, x^{*}\right], \\
C_{2} \hat{y}\left(\tau, q, \lambda_{n}\right), & \text { as } \tau \in\left(x^{*}, \pi\right],
\end{array} \quad C_{i} \neq 0, i=1,2 .\right.
$$

The relation $C_{i} \neq 0, i=1,2$, follows from the condition $x^{*}=x_{k, n}=\tilde{x}_{l, m} \in(0, \pi)$ and thus $\beta_{k, n}(\tau) \neq 0, \tilde{\beta}_{l, m}(\tau) \neq 0$. Since $\hat{y}$ and $\hat{\tilde{y}}$ solve differential equations in problem (1) for the corresponding eigenvalues $\lambda_{n}, \tilde{\lambda}_{m}$ and potentials $q$ and $\tilde{q}$, we obtain

$$
q(\tau)-\lambda_{n} \stackrel{\text { a.e. }}{=} \frac{\hat{y}^{\prime \prime}\left(\tau, q, \lambda_{n}\right)}{\hat{y}\left(\tau, q, \lambda_{n}\right)} \stackrel{\text { a.e. }}{=} \frac{\hat{\tilde{y}}^{\prime \prime}\left(\tau, \tilde{q}, \lambda_{n}\right)}{\hat{\tilde{y}}(\tau, \tilde{q}, \tilde{\lambda} m)} \stackrel{\text { a.e. }}{=} \tilde{q}(\tau)-\tilde{\lambda}_{m} .
$$

Integrating the obtain relation w.r.t. $\tau$ from 0 to $\pi$ and bearing in mind the normalization (3), we obtain $\lambda_{n}=\tilde{\lambda}_{m}$.

Identities $\tilde{\alpha}=\alpha, \tilde{\beta}=\beta$ follow from (1), (8) and (18). The proof is complete.

Proof of Proposition 4. To prove the proposition, in the proof of Theorem 5 we continue potentials $q$ and $\tilde{q}$ in relation $(19)$ by continuity and it completes the proof.

Proof of Proposition 5. In order not to overload the arguments by technical details, we provide the proof for Dirichlet case $\alpha=\pi m, \beta=\pi p, m, p \in \mathbb{Z}$.

As $\tilde{q}$ we take $\tilde{q} \equiv 0$, then $\tilde{x}_{k, n}=\frac{k \pi}{n}$ for each $n \in \mathbb{N}$ and $0 \leqslant k \leqslant n$. For an arbitrary interval $(a, b) \subset[0, \pi]$ there exist $n \in \mathbb{N}$ and $0 \leqslant k_{0} \leqslant n-1$ such that $\left[x_{k_{0}, n}, x_{k_{0}+1, n}\right] \subset(a, b)$.

Let $0 \leqslant z \leqslant t \leqslant \frac{\pi}{2 n}$. We consider the potential

$$
\breve{q}(x, z, t)= \begin{cases}\frac{2}{\left(x-x_{k_{0}, n}-t\right)^{2}-t^{2}+z^{2}}, & x_{k_{0}, n}+z \leqslant x \leqslant x_{k_{0}, n}+t, \\ \frac{2}{\left(x_{k_{0}+1, n}-x-t\right)^{2}-t^{2}+z^{2}}, & x_{k_{0}+1, n}-t \leqslant x \leqslant x_{k_{0}+1, n}-z, \\ -n^{2}, & x \in[0, \pi] \backslash\left[x_{k_{0}, n}, x_{k_{0}+1, n}\right], \\ 0, & \text { otherwise, }\end{cases}
$$

not obeying normalization condition (3).

The eigenfunction of problem (1) with potential (20) associated with $n$th eigenvalue $\breve{\lambda}_{n}=0$ reads as

$$
\begin{gathered}
y\left(x, \breve{q}, \breve{\lambda}_{n}, z, t\right)= \\
= \begin{cases}(-1)^{k_{0}} Y\left(x-x_{k_{0}, n}, z, t\right), & x_{k_{0}, n} \leqslant x \leqslant \frac{x_{k_{0}, n}+x_{k_{0}+1, n}}{2}, \\
(-1)^{k_{0}} Y\left(x_{k_{0}+1, n}-x, z, t\right), & \frac{x_{k_{0}, n}+x_{k_{0}+1, n}}{2}<x \leqslant x_{k_{0}+1, n}, \\
\sin n x, & x \in[0, \pi] \backslash\left[x_{k_{0}, n}, x_{k_{0}+1, n}\right],\end{cases}
\end{gathered}
$$

where

$$
Y(x, z, t)= \begin{cases}n x, & \text { as } x \in[0, z], \\ -\frac{n}{2(t-z)}(x-t)^{2}+n z+\frac{n(t-z)}{2}, & \text { as } x \in(z, t] \\ n z+\frac{n(t-z)}{2}, & \text { as } x \in\left(t, \frac{\pi}{2 n}\right] .\end{cases}
$$

Here the eigenfunctions of problems (1) with potentials 20 and $\tilde{q} \equiv 0$ for each $0 \leqslant z \leqslant t \leqslant \frac{\pi}{2 n}$ are both normalized by the condition $y^{\prime}\left(0, \breve{q}, \breve{\lambda}_{n}, z, t\right)=\tilde{y}^{\prime}\left(0, n^{2}\right)=n$. Moreover, the sets of their zeros $x_{k, n}=\tilde{x}_{k, n}=\frac{k \pi}{n}, 0 \leqslant k \leqslant n$, coincide. 
We consider the function

$$
\begin{aligned}
F(z, t)= & \frac{1}{2}\left\|y^{2}\left(\cdot, \breve{q}, \breve{\lambda}_{n}, z, t\right)\right\|_{L_{2}\left(x_{k_{0}, n}, x_{k_{0}+1, n}\right)}^{2}-\frac{1}{2}\left\|\tilde{y}^{2}\left(\cdot, \tilde{q}, n^{2}\right)\right\|_{L_{2}\left(x_{k_{0}, n}, x_{k_{0}+1, n}\right)}^{2} \\
& =\frac{n^{2} z^{3}}{3}+\frac{n^{2}(t-z)^{3}}{20}-\frac{n^{2}(t+z)(t-z)^{2}}{6}+\left\{\frac{n(t+z)}{2}\right\}^{2}\left(\frac{\pi}{2 n}-z\right)-\frac{\pi}{4 n} .
\end{aligned}
$$

in the triangle $0 \leqslant z \leqslant t \leqslant \frac{\pi}{2 n}$. Function $F$ is continuous in the closed domain $0 \leqslant z \leqslant t \leqslant \frac{\pi}{2 n}$. Through the points with the coordinates $z=0, t=0$ and $z=\frac{\pi}{2 n}, t=\frac{\pi}{2 n}$ we draw a continuous curve $\Gamma$ belonging to the interior (except the end-points of the curve) of the domain $0<z<$ $t<\frac{\pi}{2 n}$. Since $F(0,0)=-\frac{\pi}{4 n}$, and $F\left(\frac{\pi}{2 n}, \frac{\pi}{2 n}\right)=\frac{\pi}{4 n}\left(\frac{\pi^{2}}{6}-1\right)$, on curve $\Gamma$ there exist points with coordinates $\left(z^{*}, t^{*}\right) \in \Gamma, 0<z^{*}<t^{*}<\frac{\pi}{2 n}$ obeying (see (21)) the identity

$$
F\left(z^{*}, t^{*}\right)=\frac{1}{2}\left\|y^{2}\left(\cdot, \breve{q}, \breve{\lambda}_{n}, z^{*}, t^{*}\right)\right\|_{L_{2}\left(x_{k_{0}, n}, x_{k_{0}+1, n}\right)}^{2}-\frac{1}{2}\left\|\tilde{y}^{2}\left(\cdot, \tilde{q}, n^{2}\right)\right\|_{L_{2}\left(x_{k_{0}, n}, x_{k_{0}+1, n}\right)}^{2}=0,
$$

where $0<z^{*}<t^{*}<\frac{\pi}{2 n}$. Together with Theorem 2 and 21) it yields the relations

$$
\left\|y^{2}\left(\cdot, \breve{q}, \breve{\lambda}_{n}, z^{*}, t^{*}\right)\right\|_{L_{2}(0, \pi)}^{2}=\left\|\tilde{y}^{2}\left(\cdot, \tilde{q}, n^{2}\right)\right\|_{L_{2}(0, \pi)}^{2}, \quad \breve{\beta}_{k, n} \equiv \tilde{\beta}_{k, n} \text { on }[0, \pi], \quad 0 \leqslant k \leqslant n,
$$

and

$$
D x_{k, n}[\breve{q}, \delta[1](x)]=D \tilde{x}_{k, n}[\tilde{q}, \delta[1](x)],
$$

for each $x \in[0, \pi] \backslash\left(x_{k_{0}, n}, x_{k_{0}+1, n}\right), 0 \leqslant k \leqslant n$.

It implies (12).

After the renormalization of the potential

$$
\breve{q}\left(x, z^{*}, t^{*}\right)= \begin{cases}\frac{2}{\left(x-x_{k_{0}, n}-t^{*}\right)^{2}-t^{* 2}+z^{* 2}}, & x_{k_{0}, n}+z^{*} \leqslant x \leqslant x_{k_{0}, n}+t^{*}, \\ \frac{2}{\left(x_{k_{0}+1, n}-x-t^{*}\right)^{2}-t^{* 2}+z^{* 2}}, & x_{k_{0}+1, n}-t^{*} \leqslant x \leqslant x_{k_{0}+1, n}-z^{*}, \\ -n^{2}, & x \in[0, \pi] \backslash\left[x_{k_{0}, n}, x_{k_{0}+1, n}\right], \\ 0, & \text { otherwise, }\end{cases}
$$

we obtain the potential satisfying (3), (11) and (12):

$$
q(x)=\breve{q}\left(x, z^{*}, t^{*}\right)-\frac{1}{\pi} \int_{0}^{\pi} \breve{q}\left(x, z^{*}, t^{*}\right) d x .
$$

Since the function $\left(x-x_{k_{0}, n}-t^{*}\right)^{2}-t^{* 2}+z^{* 2}$ is separated from zero on the segment $x_{k_{0}, n}+z^{*} \leqslant$ $x \leqslant x_{k_{0}, n}+t^{*}$ and the same is true for the function $\left(x_{k_{0}+1, n}-x-t^{*}\right)^{2}-t^{* 2}+z^{* 2}$ on the segment $x_{k_{0}+1, n}-t^{*} \leqslant x \leqslant x_{k_{0}+1, n}-z^{*}$, potential $q$ is a function with bounded variation on $[0, \pi]$. By (21) all the zeroes of eigenfunctions $y\left(\cdot, q, \lambda_{n}, z^{*}, t^{*}\right)$ and $\tilde{y}\left(\cdot, \tilde{q}, n^{2}\right)$ coincide.

The case of arbitrary $\alpha, \beta \in \mathbb{R}$ can be proven in the same way. The proof is complete.

Remark. Since curve $\Gamma$ in the proof of Proposition 5 was chosen in arbitrary way in the interior of domain $0<z<t<\frac{\pi}{2 n}$, then the amount of various points $\left(t^{*}, z^{*}\right)$ and thus of various potentials $q$ satisfying conditions (11), (12) of Proposition 5 is at least continuum.

\section{BIBLIOGRAPHY}

1. V.A. Vinokurov, V.A. Sadovnichii. Asymptotics of any order for the eigenvalues and eigenfunctions of the Sturm-Liouville boundary-value problem on a segment with a summable potential // Izv. RAN. Ser. matem. 2000. V. 64, No. 4. P. 47-108. [Izv. Math. 2000. V. 64, No. 4. P. 695-754.]

2. A.M. Savchuk, A.A. Shkalikov. Sturm-Liouville operators with singular potentials // Matem. Zametki. 1999. V. 66, No. 6. P. 897-912. [Math. Notes. 1999. V. 66, No. 6. P. 741-753.]

3. A.M. Savchuk. On the eigenvalues and eigenfunctions of the Sturm-Liouville operator with a singular potential // Matem. Zametki. 2001. V. 69, No. 2. P. 277-285. [Math. Notes. V. 69, No. 2. P. 245-252.] 
4. A.M. Savchuk, A.A. Shkalikov. On the properties of maps connected with inverse Sturm-Liouville problems // Trudy Inst. Matem. V.A. Steklova. 2008. V. 260. P. 227-247. [Proc. Steklov Inst. Math. 2008. V. 260, No. 1. P. 218-237.]

5. A.M. Savchuk, A.A. Shkalikov. On the eigenvalues of the Sturm-Liouville operator with potentials from Sobolev spaces // Matem. Zametki. 2006. V. 80, No. 6. P. 897-912. [Math. Notes. 2006. V. 80, No. 6. P. 814-832.]

6. A.A. Shkalikov, O.A. Veliev. On the Riesz basis property of the eigen- and associated functions of periodic and antiperiodic Sturm-Liouville problems // Matem. Zametki. 2009. V. 85, No. 5. P. 671-686. [Math. Notes. 2009. V. 85, No. 5-6. P. 647-660.]

7. S.P. Suetin. Spectral properties of a class of discrete Sturm-Liouville operators // Uspekhi Matem. Nauk. 2006. V. 61, No. 2(368). P. 171-172. [Russ. Math. Surv. 2006. V. 61, No. 2. P. 365-367.]

8. D.I. Borisov, R.R. Gadyl'shin. On spectrum of periodic operator with a small localized perturbation // Izv. RAN. Ser. matem. 2008. V. 72, No. 4. P. 37-66. [Izv. Math. 2008. V. 72, No. 4. P. 659-688.]

9. D.I. Borisov, R.R. Gadyl'shin. On spectrum of self-adjoint differential operator on the axis with fast oscillating coefficients // Matem. Sbornik. 2007. V. 198, No. 8. P. 3-34. [Sb. Math. 2007. V. 198, No. 8. P. 1063-1093.]

10. D.I. Borisov. Some singular perturbations of periodic operators // Teor. Matem. Fiz. 2007. V. 151. No. 2. P. 207-218. [Theor. Math. Phys. 2007. V. 151. No. 2. P. 614-624.]

11. Yu.V. Pokornyi, V.L. Pryadiev. Some problems of the qualitative Sturm-Liouville theory on a spatial network // Uspekhi Matem. Nauk. 2004. V. 59, No. 3(357). P. 116-150. [Russ. Math. Surv. V. 59, No. 3. P. 515-552.]

12. Yu.V. Pokornyi, M.B. Zvereva, A.S. Ishchenko, S.A. Shabrov. An irregular extension of the oscillation theory of the Sturm-Liouville spectral problem // Matem. Zametki. 2007. V. 82, No. 4. P. 578-582. [Math. Notes. 2007. V. 82, No. 3-4. P. 518-521.]

13. Yu.V. Pokornyi, M.B. Zvereva, S.A. Shabrov. Sturm-Liouville oscillation theory for impulsive problems // Uspekhi matem. nauk. 2008. V. 63, No. P. 111-154. [Russ. Math. Surv. 2008. V. 63, No. P. 109-153.]

14. A.Yu. Trynin. A generalization of the Whittaker-Kotel'nikov-Shannon sampling theorem for continuous functions on a closed interval // Matem. Sbornik. 2009. V. 200, No. 11. P. 61-108. [Sb. Math. 2009. V. 200, No. 11. P. 1633-1679.]

15. A.Yu. Trynin. Asymptotic behavior of the solutions and nodal points of Sturm-Liouville differential expressions // Sibir. Matem. Zhurn. 2010. V. 51, No. 3. P. 662-675. [Sib. Math. J. 2010. V. 51, No. 3. P. 525-536.]

16. V. Ambarzumian. Über eine Frage der Eigenwerttheorie // Zeitschr. für Physik. 1929. V. 53, No. 9-10. P. 690-695.

17. G. Borg. Eine Umkehrung der Sturm-Liouvillschen Eigenwertaufgabe // Acta Math. 1946. V. 78, No. 1. P. 1-96.

18. N. Levinson. On the uniqueness of the potential in a Schrödinger equation for a given asymptotic phase // Danske Vid. Selsk. Mat. Fys. Medd. 1949. V. 25, No. 9. P. 25.

19. L.A. Chudov. The inverse Sturm-Liouville problem// Matem. Sbornik. 1949. V. 25(67), No. 3. P. 451-454. (in Russian.)

20. V.A. Marchenko. Some question of theory of second order differential operator // Dokl. Akad. Nauk SSSR. 1950. V. 72, No. 3. P. 457-460.

21. I.M. Gel'fand, B.M. Levitan. On the determination of a differential equation from its spectral function // Izv. Akad. Nauk. SSSR. Ser. matem. 1951. V. 15, No. 4. P. 309-360. (in Russian.)

22. V.A. Marchenko. Some questions of the theory of one-dimensional linear differential operators of the second order. I // Trudy Mosk. Matem. Obsch. 1952. V. 1. P. 327-420. (in Russian.)

23. B.M. Levitan. On the determination of the Sturm-Liouville operator from one and two spectra // Izv. Akad. Nauk SSSR. Ser. matem. 1978. V. 42, No. 1. P. 185-199. [Math. USSR. Izv. 1978. V. 12, No. 1. P. 179-193.]

24. B.M. Levitan. Inverse problem for the Sturm-Liouville operator in the case of finite-range and infinite-range potentials // Trudy Mosk. Matem. Obsch. 1982. V. 45. P. 3-36. (in Russian.) 
25. A.M. Savchuk, A.A. Shkalikov. Inverse problems for Sturm-Liouville operators with potentials in Sobolev spaces: Uniform stability // Funkt. Anal. Pril. 2010. V. 44, No. 4. P. 34-53. [Funct. Anal. Appl. 2010. V. 44, No. 4. P. 270-285.]

26. M.I. Belishev. Boundary control and inverse problems: The one-dimensional variant of the BCmethod // Zapiski Nauch. Semin. POMI. 2008. V. 354. P. 19-80. [J. Math. Sci. 2008. V. 155, No. 3. P. 343-378.]

27. J.R. McLaughlin. Inverse spectral theory using nodal points as data - a uniqueness result // J. Differ. Equat. 1988. V. 73, No. 2. P. 354-362.

28. E.S. Panakhov, H. Koyunbakan. Inverse nodal problems for second order differential operators with a regular singularity // Int. J. Difference Equat. 2006. V. 1, No. 2. P. 241-247.

29. Y-H. Cheng, C.K. Law, J. Tsay. Remarks on a new inverse nodal problem // J. Math. Anal. Appl. 2000. V. 248, No. 1. P. 145-155.

30. Sh. Akbarpoor, A.H. Dabbaghian. The uniqueness theorem for boundary value problem with aftereffect and eigenvalue in the boundary condition // Int. J. Contemp. Math. Sciences. 2011. V. 6, No. 20. P. 963-970.

31. M. Sat, E.S. Panakhov. Inverse nodal problem for Sturm-Liouville operators with coulomb potential // Int. J. Pure Appl. Math. 2012. V. 80, No. 2. P. 173-180.

32. V.A. Yurko. Inverse nodal problems for the Sturm-Liouville differential operators on a star-type graph // Sibir. Matem. Zhurn. 2009. V. 50, No. 2. P. 469-475. [Sib. Math. J. 2009. V. 50, No. 2. P. 373-378.]

33. V.A. Marchenko. Sturm-Liouville operators and their applications. Naukova Dumka, Kiev, 1977. (in Russian.)

34. B.M. Levitan. Inverse Sturm-Liouville problems. Nauka, Moscow, 1984. [VNU Science Press, Utrecht, 1987.]

35. B.M. Levitan, I.S. Sargsyan. Sturm-Liouville and Dirac operators. Nauka, Moscow, 1988. [Kluwer Academic Publishers, Dordrecht, 1990.]

36. A.Yu. Trynin. On the absence of stability of interpolation in eigenfunctions of the Sturm-Liouville problem // Izv. Vysshikh Uchebnykh Zavedenij. 2000. No. 9(460). P. 60-73. [Russ. Math. Izvestiya VUZ. Matematika. 2000. V. 44, No. 9. P. 58-71.]

37. A.Yu. Trynin. Differential properties of the zeros the eigenfunctions Sturm Liouville problems // Ufimskij Matem. Zhurn. 2011. V. 3, No. 4. P. 133-143. [Ufa Math. J. 2011. V. 3, No. 4. P. 130-140.]

38. V.S. Vladimirov. Equations of mathematical physics. Nauka, Moscow, 1988. [1st ed. Marcel Dekker, New York, 1971.]

39. S.M. Nikolsky. A course of mathematical analysis. V. 2. Nauka, Moscow, 1983. [Mir Publishers, Moscow, 1987.]

40. P. Hartman. Ordinary differential equations. John Wiley \& Sons, New York, 1964.

Alexander Yurievich Trynin,

Saratov State University, Astrakhanskaya str., 83, 410012, Saratov, Russia

E-mail: atrynin@gmail.com 